\title{
Safety and diagnostic performance of pulmonologists performing electromagnetic guided percutaneous lung biopsy (SPiNperc)
}

\author{
Christopher MALloW, ${ }^{1}$ (D) Hans LeE, ${ }^{1}$ Catherine OBERG ${ }^{2}$ Jeffrey THIBOUtot, ${ }^{1}$ Jason AKUlian, ${ }^{3}$ \\ Allen C. BuRKS, ${ }^{4}$ (i) Branden LUNA, ${ }^{5}$ Sadia Benzaquen, ${ }^{6}$ Hitesh BATRA, ${ }^{7}$ Jose CARdenAS-GARCiA, ${ }^{8}$ \\ JenNifER TOTH ${ }^{9}$ JAY HEIDECKER,${ }^{10}$ AdAM BELANGER, ${ }^{3}$ JASON MCCLUNE, ${ }^{9}$ UMAR OSMAN, ${ }^{11}$ \\ Venkatesh lakshminaraYANAN, ${ }^{12}$ Nicholas PASTIS, ${ }^{5}$ Gerard SILVESTRI, ${ }^{5}$ AleXander CHEN $^{4}$ \\ AND LONNY YARMUS ${ }^{1}$ (D)
}

\begin{abstract}
${ }^{1}$ Section of Interventional Pulmonology, Division of Pulmonary and Critical Care Medicine, Johns Hopkins University School of Medicine, Baltimore, MD, USA; ${ }^{2}$ Division of Pulmonary and Critical Care Medicine, Ichan School of Medicine at Mount Sinai, New York, NY, USA; ${ }^{3}$ Section of Interventional Pulmonology, Division of Pulmonary and Critical Care Medicine, University of North Carolina School of Medicine, Chapel Hill, NC, USA; ${ }^{4}$ Section of Interventional Pulmonology, Division of Pulmonary and Critical Care Medicine, Washington University in St. Louis School of Medicine, St. Louis, MO, USA; ${ }^{5}$ Division of Pulmonary and Critical Care Medicine, Medical University of South Carolina, Charleston, SC, USA; ${ }^{6}$ Section of Interventional Pulmonology, Division of Pulmonary and Critical Care Medicine, University of Cincinnati College of Medicine, Cincinnati, OH, USA;

${ }^{7}$ Section of Interventional Pulmonology, Division of Pulmonary and Critical Care Medicine, University of Alabama at Birmingham School of Medicine, Birmingham, AL, USA; ${ }^{8}$ Division of Pulmonary and Critical Care Medicine, University of Michigan School, Ann Arbor, MI, USA; ${ }^{9}$ Section of Interventional Pulmonology, Division of Pulmonary and Critical Care Medicine, Penn State Health Milton S. Hershey Medical Center, Hershey, PA, USA; ${ }^{10}$ Division of Pulmonary Medicine, Birmingham Pulmonary Group, Birmingham, AL, USA; ${ }^{11}$ Division of Pulmonary and Critical Care Medicine, Memorial Health System, Marietta, OH, USA; ${ }^{12}$ Division of Pulmonary and Critical Care Medicine, Pulmonary and Critical Care of Atlanta, Atlanta, GA, USA
\end{abstract}

\begin{abstract}
Background and objective: Percutaneous lung biopsy for diagnostic sampling of peripheral lung nodules has been widely performed by interventional radiologists under computed tomography (CT) guidance. New technology allows pulmonologists to perform percutaneous lung biopsies using electromagnetic (EM) guided technology. With the adoption of this new technique, the safety, feasibility and diagnostic yield need to be explored. The goal of this study was to determine the safety, feasibility and diagnostic yield of EM-guided percutaneous lung biopsy performed by pulmonologists. Methods: We conducted a retrospective, multicentre study of 129 EM-guided percutaneous lung biopsies that occurred between November 2013 and March 2017. The study consisted of seven academic and three community medical centres.

Results: The average age of participants was 65.6 years, BMI was 26.3 and $50.4 \%$ were females. The majority of lesions were in the right upper lobe $(37.2 \%)$ and left upper lobe $(\mathbf{3 1 . 8 \%})$. The mean size of the lesions was $27.31 \mathrm{~mm}$ and the average distance from the pleura was $13.2 \mathrm{~mm}$. Practitioners averaged two fine-needle aspirates and five core biopsies per procedure. There were
\end{abstract}

Correspondence: Lonny Yarmus, Division of Pulmonary and Critical Care Medicine, Johns Hopkins University School of Medicine, 1830 East Monument Street, 5th Floor, Baltimore, MD 21287, USA. Email: Iyarmus@jhmi.edu

Received 17 July 2018; invited to revise 24 August and 25 November 2018; revised 3 and 28 November 2018; accepted 10 December 2018 (Associate Editor: Pyng Lee; Senior Editor: Phan Nguyen).

\section{SUMMARY AT A GLANCE}

Lung cancer screening has led to the discovery of over 1 million pulmonary nodules each year. New technology allows pulmonologists to perform percutaneous lung biopsies using electromagnetic (EM) guided technology. In this retrospective analysis, we demonstrate that EM percutaneous needle biopsy is safe, feasible and provides an acceptable diagnostic yield.

23 (17.8\%) pneumothoraces, of which 16 (12.4\%) received small-bore chest tube placement. The diagnostic yield of percutaneous lung biopsy was $73.7 \%$. When EM-guided bronchoscopic sampling was also performed during the same procedural encounter, the overall diagnostic yield increased to $81.1 \%$.

Conclusion: In this large multicentred series, the use of EM guidance for percutaneous lung biopsies was safe and feasible, with acceptable diagnostic yield in the hands of pulmonologists. A prospective multicentre trial to validate these findings is currently underway (NCT03338049).

Key words: lung cancer, bronchoscopy and interventional techniques, pneumothorax, pathology.

\section{INTRODUCTION}

The National Lung Screening Trial (NLST) showed a $20 \%$ mortality decrease when using low-dose computed 
tomography (CT) screening compared to conventional radiology for the detection of lung cancer. ${ }^{1}$ With over 8 million Americans meeting the criteria for lung cancer screening, ${ }^{2}$ accurate and rapid diagnosis of suspicious solitary pulmonary nodules (SPN) is needed. There are over 10 million chest CT scans performed each year in the United States in which an estimated 1.5 million nodules are discovered..$^{3-5}$ This number is expected to increase given the endorsement of lowdose CT scans for lung cancer screening by the US Preventative Services Task Force (USPSTF). ${ }^{1,6}$ Successful diagnosis of an early stage lung cancer gives an opportunity to cure patients of the disease; however, the majority of nodules biopsied will be benign and thus improved minimally invasive techniques are needed to help reduce unnecessary surgery. ${ }^{7}$

The current common procedures used to diagnose SPN are surgical biopsy, percutaneous lung biopsy and bronchoscopy. ${ }^{8}$ Surgical biopsies have a high diagnostic yield, but at the cost of undergoing a more invasive and lengthy procedure under general anaesthesia and a higher morbidity than either of the other two options. Typically, interventional radiologists perform percutaneous lung biopsies under CT guidance, often performing multiple pleural punctures to make a diagnosis in real time. Although diagnostic yield is higher than bronchoscopy alone, this comes at the cost of higher procedural complications. A large population-based study of over 15000 patients found a $16 \%$ pneumothorax rate and a $1 \%$ risk of major haemorrhage in patients undergoing a CT-guided lung biopsy. ${ }^{9}$ Although the bronchoscopic yield for SPN historically remains low, some advantages to bronchoscopy include a lower complication rate $^{10}$ as well as the ability to stage the disease at the time of diagnosis with mediastinal and hilar sampling using endobronchial ultrasound (EBUS). ${ }^{11}$

A recent technological advancement, electromagnetic (EM) guided percutaneous lung biopsy, has become available. The system allows clinicians to biopsy an SPN by performing a single percutaneous pass through the visceral pleural under EM guidance alone without the need for real-time CT or fluoroscopic guidance. The procedure is performed under EM guidance that is linked to a CT scan obtained just prior to the procedure.

This platform provides proceduralists the novel capability of performing an algorithmic approach to the procedure to potentially optimize yield and decrease procedural complications by performing EBUS for lymph node staging first in the presence of rapid on-site evaluation (ROSE) and if there is no evidence of nodal involvement, under the same procedural setting, attempt an EM-guided bronchoscopy. The EM-guided bronchoscopy and EM percutaneous lung biopsy can be performed using the same software. If a diagnosis is unachievable with a bronchoscopic approach, the proceduralist can pivot again to perform an EM-guided percutaneous lung biopsy during the same procedural setting. This procedure may take place in a procedure suite or operating room, and adds approximately $20 \mathrm{~min}$ to the total procedure time. $^{12}$
The goal of this study was to assess the safety, feasibility and diagnostic yield of pulmonologists performing an EM-guided percutaneous lung biopsy.

\section{METHODS}

This was a multicentre, retrospective study on pulmonologist performed EM percutaneous lung biopsies from November 2013 through March 2017. Institutional Review Board approval with waiver of consent was obtained from each of the 10 individual institutions (Institutional Review Board 00116317) prior to initiation of data collection. All patients who received an EM percutaneous lung biopsy were identified using CPT codes (32405 and 10022) and were included in the data analysis. All procedures were performed using the Veran SPiNperc EM guidance system (Veran Medical, St Louis, MO, USA). The outcomes for this study included safety, feasibility and diagnostic yield of this procedure.

All patients were referred to pulmonology for a lung lesion suspicious for cancer, and were scheduled to undergo EM navigational bronchoscopy and EMguided percutaneous lung biopsy. All patients received a chest CT scan on the day of the procedure with $0.5 \mathrm{~mm}$ intervals and $0.67-0.75 \mathrm{~mm}$ thickness, with navigational tracking pads (vPAD2, Veran Medical) in place which leads to the creation of a trackable virtual airway map (Veran Medical). Pre-procedure, using the software platform, the physician performing the procedure identified and marked the target lesion and determined where on the chest wall the needle would be placed in order to percutaneously access the lesion. The software then provided the site of entry and trajectory of the needle during real-time EM guidance for the percutaneous approach. All patients were placed under moderate or deep sedation for the bronchoscopy, which was continued throughout the duration of the procedure if EM-guided percutaneous needle biopsy was performed. An EM tip tracked biopsy needle introducer was used under navigational guidance to identify the previously determined entry site. Upon localization of the nodule, an introducer needle was passed through the visceral pleura and a 20-gauge Chiba needle used for fine-needle aspiration followed by a 20-gauge coaxial core biopsy instrument (SuperCore Argon Medical, Frisco, TX, USA) was utilized through the introducer needle to obtain tissue samples. Upon completion of the lung biopsy, the introducer needle was removed and a bandage was placed. The complete details of this procedure have been previously described and are illustrated in Figure $1 .{ }^{12}$ Upon completion of the procedure, all patients were evaluated by chest X-ray or thoracic ultrasound for pneumothorax.

\section{Data analysis}

Demographic information, including age, sex, race, smoking history and history of lung disease, was collected. Characteristics of the biopsied nodule including location, size, morphology, distance from the pleura and Positron emission tomography scan avidity were collected. The number of tissue samples, adverse events and diagnostics were recorded when available. 
(a)

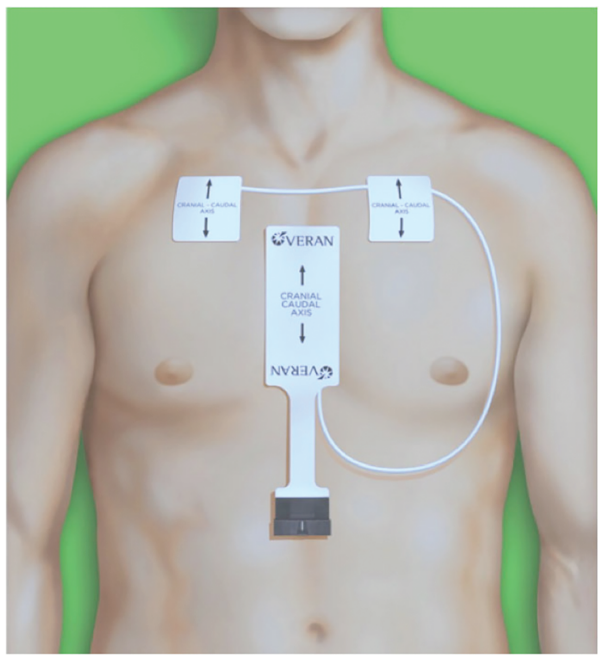

(b)

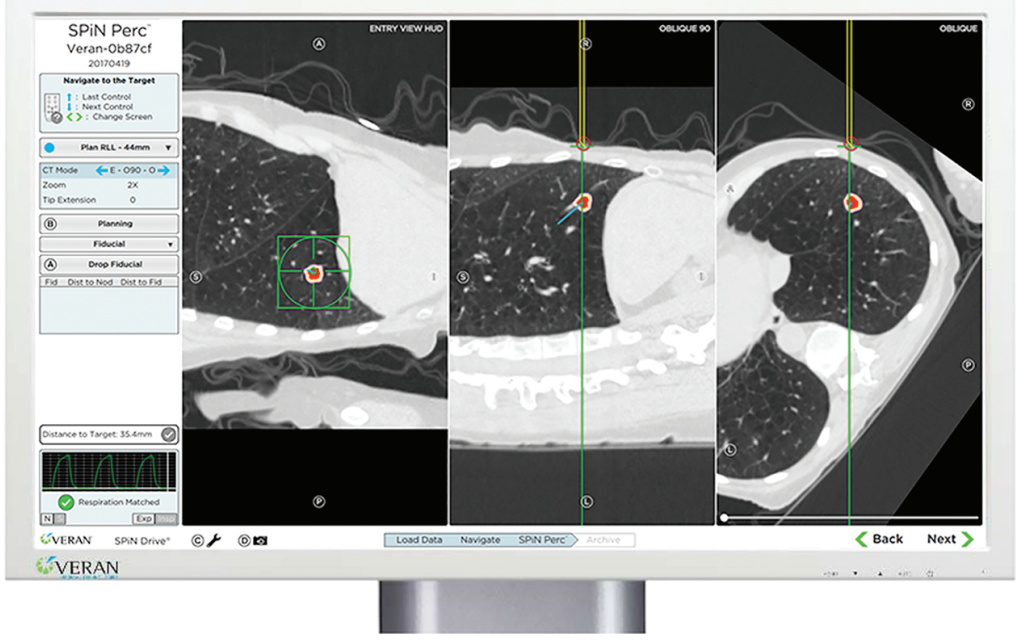

Figure 1 Electromagnetic (EM) navigational trackpad placement and percutaneous lung biopsy planning programme. The panel on the left shows the placement of the tracking pads needed prior to computed tomography (CT) scan to utilize the EM navigational software. These pads can be shifted to the left or the right in order to clear access for the percutaneous lung biopsy. The panel on the right shows the planning screen used prior to performing the transcutaneous needle biopsy. The programme will identify the nodule, which can be optimized by the user, followed by placement of a virtual percutaneous needle. The placement of the needle aids the user in locating the optimal site to perform the percutaneous needle biopsy.

Additional adverse event data were collected, including rates of pneumothorax, site of bleeding, haemoptysis, infection and post-procedure admission. Diagnostic yield data, including adequacy of sample, EM-guided percutaneous lung biopsy and FNA yield, if another procedure was performed and associated yield and final diagnosis were obtained. A biopsy was deemed diagnostic only if there was a definitive neoplastic diagnosis (adenocarcinoma, small cell carcinoma, etc.) or a definitive benign diagnosis (fibrosis, granuloma, etc.) with confirmed radiographic stability up to 1 ear. Cellular atypia or lung parenchyma without a confirmed pathologic diagnosis, with or without radiographic stability, was considered incomplete in the final analysis. All patients with incomplete data were excluded from the diagnostic analysis of the statistics.

A large proportion of participants were obtained from a single centre. Because of this, we conducted a secondary analysis comparing this site to the remaining sites pertaining to diagnostic yield and adverse event data. Continuous variables are described using means with SD or range. Dichotomous variables are summarized as simple proportions. Simple t-tests were used to compare populations when necessary. Logistic regression was used to identify potential risk factors for both adverse events and diagnostic yield. A $P$-value of $<0.05$ was considered statistically significant. All statistics were performed using STATA 14.2 (StataCorp LLC, College Station, TX, USA).

\section{RESULTS}

There were 129 patients who received EM-guided percutaneous lung biopsies identified at 10 centres. A consort diagram can be found in Figure 2. Of these centres, seven were academic sites and three

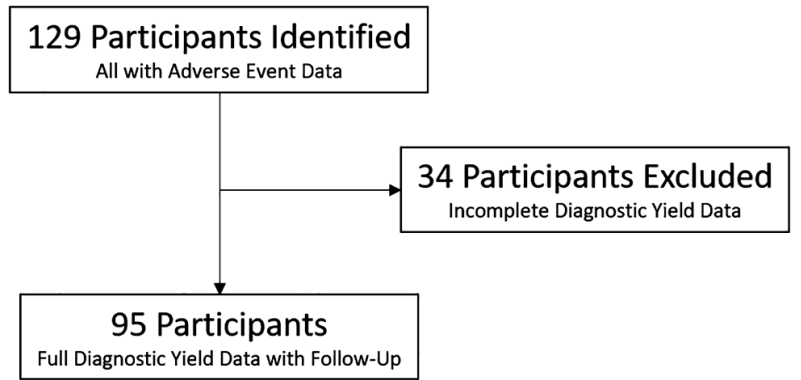

Figure 2 Patient recruitment flow diagram.

community sites. The average age of participants was 65.6 years, and half were females. The baseline characteristics are shown in Table 1.

The characteristics of the lesions sampled are shown in Table 2. The majority of lesions were located in the upper lobes. There was a mean of one pass of the introducer needle into the target (range: 1-3) performed per patient with the introducer needle. There were two FNA (range: $0-7$ ) and five core (range: $0-10$ ) biopsies taken.

Adverse event data are presented in Table 3. The location of the nodule biopsied was not an independent risk factor for an adverse event (OR: 0.87; 95\% CI: $0.67,1.13$ ). Given these findings, it was excluded from the multivariable analysis. There was no association between the presence of an adverse event and size of the lesion (OR: 0.97; 95\% CI: 0.94, 1.01; for each $1 \mathrm{~mm}$ increase in nodule size). There was a trend towards increased risk of adverse events seen with increasing distance from the pleura (OR: 1.02; 95\% CI: 0.99, 1.05; for each $1 \mathrm{~mm}$ increase in nodule size). The presence 
Table 1 Baseline characteristics

\begin{tabular}{lc}
\hline Characteristic & All patients \\
\hline Age, mean (range) & $65.52(27-91)$ \\
Female sex (\%) & 50.40 \\
BMI, mean (SD) & $26.30(5.97)$ \\
Race (\%) & \\
$\quad$ White & 61.24 \\
$\quad$ Black & 18.60 \\
Smoking history & \\
Current (\%) & 31.78 \\
Prior (\%) & 51.16 \\
$\quad$ Never (\%) & 17.05 \\
$\quad$ Pack-years, mean (SD) & $28.0(23.56)$ \\
History of COPD (\%) & 38.76 \\
History of emphysema on CT (\%) & 55.04 \\
\hline
\end{tabular}

CT, computed tomography.

Table 2 Lung nodule characteristics

\begin{tabular}{lc}
\hline Characteristics & All patients \\
\hline Location (\%) & \\
Right upper lobe & 37.21 \\
Right middle lobe & 10.85 \\
Right lower lobe & 7.75 \\
Left upper lobe & 31.78 \\
Left lower lobe & 7.75 \\
Lingula & 4.65 \\
Size (mm), mean (range) & $27.31(9-140)$ \\
Distance from the pleura (mm), mean & $13.20(0-63)$ \\
(range) & \\
\hline
\end{tabular}

Table 3 Adverse events

\begin{tabular}{lc}
\hline Adverse event & $\begin{array}{c}\text { Percentage of } \\
\text { patients }(n=129)\end{array}$ \\
\hline Overall & $29(22.5 \%)$ \\
Pneumothorax & $23(17.8 \%)$ \\
$\quad$ Requiring chest tube $\quad$ placement & $16(12.4 \%)$ \\
Minor bleeding & $2(1.6 \%)$ \\
COPD exacerbation & $1(0.78 \%)$ \\
Transient hypercapnia & $2(1.6 \%)$ \\
Transient hypoxaemia & $1(0.78 \%)$
\end{tabular}

of emphysema on CT scan, history of COPD and number of pack-years smoking also did not lead to increased odds of an adverse event. As the size of lesion increased, there was a decreased risk of pneumothorax (OR: 0.95; 95\% CI: 0.90, 1.01; for each $1 \mathrm{~mm}$ increase in nodule size). There was no association between the number of pleural punctures with the introducer needle and risk of pneumothorax. There was also no association between the number of core biopsies, or FNA, and the risk of an adverse event.

Ninety-five participants had complete data reporting available for diagnostic yield, after excluding patients
Table 4 Diagnoses obtained, electromagnetic percutaneous diagnosis versus all diagnoses

\begin{tabular}{lcc}
\hline Diagnosis & $\begin{array}{c}\text { Percutaneous } \\
\text { diagnosis } \\
(n=70)\end{array}$ & $\begin{array}{c}\text { All } \\
\text { diagnoses } \\
(n=95)\end{array}$ \\
\hline Malignant & & \\
$\quad$ Adenocarcinoma & $23(31.9 \%)$ & $32(33.7 \%)$ \\
Squamous cell & $13(18.1 \%)$ & $20(21.1 \%)$ \\
$\quad$ carcinoma & $3(4.2 \%)$ & $3(3.2 \%)$ \\
Neuroendocrine & $2(2.8 \%)$ & $2(2.1 \%)$ \\
Sarcomatoid carcinoma & $1(1.4 \%)$ & $1(1.1 \%)$ \\
Hurthle cell & $1(1.4 \%)$ & $1(1.1 \%)$ \\
NSCLC, undifferentiated & $1(1.4 \%)$ & $4(4.2 \%)$ \\
Metastases & 0 & $1(1.1 \%)$ \\
SCLC & & \\
Benign & $6(8.3 \%)$ & $6(6.3 \%)$ \\
Fibrosis & $6(8.3 \%)$ & $8(8.4 \%)$ \\
Granuloma & $5(6.9 \%)$ & $5(5.3 \%)$ \\
COP & $5(6.9 \%)$ & $5(5.3 \%)$ \\
Inflammatory & $3(4.2 \%)$ & $5(5.3 \%)$ \\
Infection & $1(1.4 \%)$ & $2(2.1 \%)$ \\
Hamartoma &
\end{tabular}

NSCLC, Non-Small Cell Lung Cancer; SCLC, Small Cell Lung Cancer.

with incomplete data as defined in the methods. The overall diagnostic yield of EM-guided percutaneous lung biopsy was $73.7 \%$. A detailed list of diagnoses can be found in Table 4 . There was no association between diagnostic yield using EM-guided percutaneous needle biopsy and lesion size (OR: 1.03; 95\% CI: $0.99,1.08$ ) which remained insignificant when analysed as a categorical variable. There was also no association in diagnostic yield and distance from the pleura (OR: 0.99; 95\% CI: 0.96, 1.02), anatomical location (OR: 0.92; 95\% CI: $0.69,1.22$ ), number of punctures (OR: 1.80; 95\% CI: $0.43,7.44)$, number of fine-needle aspirations (OR: 1.06 ; $95 \%$ CI: $0.70,1.59$ ) or the number of core biopsies obtained (OR: 0.78; 95\% CI: 0.57, 1.08). When the lesion was abutting the pleura, there was a diagnostic yield of $85 \%$ and a lower pneumothorax rate of $10 \%$. Patients who developed a pneumothorax also had an overall lower diagnostic rate of $63 \%$. When EMguided bronchoscopic sampling was also performed during the same procedural encounter, the overall diagnostic yield increased to $81.1 \%$.

Fifty-one percent of all procedures were conducted at a single site. When comparing this site to the rest of the locations, where 10 or less cases were performed at each site, there was a higher yield and lower complication rate, although these results did not reach statistical significance. The single site had an $18.1 \%$ adverse event rate versus $27 \%$ at the remainder of the sites $(P=0.23)$. The single site also had a $15.1 \%$ pneumothorax rate versus $20.6 \%$ at the remaining sites $(P=0.42)$. Of the patients who had a pneumothorax, $7.6 \%$ received chest tubes at the single site, while $17.5 \%$ received chest tubes at the remaining sites $(P=0.09)$. There was also a diagnostic rate of $78.4 \%$ at the single centre versus $68.1 \%$ at the remaining sites $(P=0.26)$. 


\section{DISCUSSION}

Until the introduction of EM-guided percutaneous lung biopsy, the routine options for percutaneous lung biopsies were performed with CT or ultrasound guidance. CT guidance has been the gold standard for the minimally invasive diagnosis of SPN with a high diagnostic yield but also a high complication rate when compared to bronchoscopy. ${ }^{11,13-15}$ Complication rates of $38.8 \%$ for core biopsies and $24 \%$ for FNA from radiologists performing CT-guided percutaneous lung biopsy were reported in a recent meta-analysis. ${ }^{16}$ Another new approach to diagnosing these lesions includes bronchoscopic transparenchymal biopsies, in which pilot studies have shown to have a diagnostic yield of $83 \%$, although with a higher pneumothorax rate (up to 33\%). ${ }^{17,18}$ A combined procedural approach for the diagnosis of pulmonary nodules by attempting a bronchoscopic diagnosis first to limit complications, followed by a percutaneous biopsy if the bronchoscopy is non-diagnostic, may be effective in limiting overall complications and increasing diagnostic yield. However, the feasibility to attempt a combined approach has been limited as few centres have a CT scanner within the bronchoscopy suite and lesions must abut the pleura to allow for adequate visualization under ultrasound guidance. ${ }^{19}$ In this study, we show that EMguided percutaneous lung biopsy was feasible with an acceptable safety profile in the hands of pulmonologists, with diagnostic yields approaching CT-guided biopsies.

We did not identify an association between the number of pleural punctures with the introducer needle and risk of pneumothorax. This is likely due to the low number of participants $(n=11)$ who received greater than one pleural puncture. Of these 11, 3 (27\%) suffered a pneumothorax in comparison to the rest of the participants in the study who only received one pleural puncture, with a $17.8 \%$ pneumothorax rate. The diagnostic rate of patients who had a pneumothorax was lower, at $63 \%$. This is likely because the pneumothorax would lead to a shift in the nodule, which would likely not be accurately tracked by the software. Similar drops in diagnostic yield are reported in the CT-guided literature.

Until the implementation of EM-guided percutaneous lung biopsy, the only options for percutaneous lung biopsies were CT-guided and ultrasound-guided. CT-guided lung biopsy has been the standard for minimally invasive diagnosis of SPN, and has been shown to have a higher diagnostic yield than bronchoscopy alone. ${ }^{11,13-15}$ We have shown that EM-guided percutaneous needle biopsy is not only safe, but also has a diagnostic yield that may approach CT-guided percutaneous needle biopsies. Combining the use of EMguided percutaneous lung biopsy with EM navigational bronchoscopy increased our diagnostic yield to $81.1 \%$. Conceptually, this not only provides overall improved diagnostic yield over bronchoscopy alone, but also leads to a decrease in overall pneumothorax rates by limiting the percutaneous approach to only those cases where ROSE is non-diagnostic for the bronchoscopic phase of the procedure. Further increases in yield and decreases in complications may also be seen with integration of EBUS with ROSE as the initial procedure but prospective validation is warranted.

Practitioner training is another important aspect of all procedures. With proper training on how to use the navigational software and equipment, coupled with expert teaching, competency in EM-guided percutaneous lung biopsy significantly increases. ${ }^{20}$ This is demonstrated by our analysis showing that experts, who performed at a single centre with the highest volume of EM-guided percutaneous lung biopsies, had a higher diagnostic rate and lower complication rate than their counterparts. By undergoing appropriate training, the provider is able to better manipulate the angle of entry into the lesion, interpret the respiratory variation and limit their technique to a single pleural puncture.

There are limitations to this study. The study was retrospective, limiting us to data available by chart review. In addition, patients were identified by CPT codes showing that a patient received an EM percutaneous lung biopsy; this referral bias was not controlled for. Although this was a multicentre retrospective chart review, many of the physicians had limited experience with EM-guided percutaneous lung biopsy. All but one site performed under 10 procedures, and $51.2 \%$ of the participants included were seen at a single large academic centre, which also limits the generalizability. This highest volume single centre had a lower pneumothorax rate, $15 \%$ ( $7.6 \%$ chest tube rate), as well as a higher diagnostic yield (78.4\%), although not statistically different from the pooled diagnostic yield and pneumothorax rate from the remaining centres. This leads us to believe that as centres perform more EMguided percutaneous lung biopsies, there will be increased safety and diagnostic yield. In addition, 32\% of the lesions diagnosed were confirmed benign thus reducing potential surgical complications by limiting benign resection rates. Lastly, we only received complete diagnostic data for 95 (74\%) subjects. Given the retrospective nature of this study, it was difficult to obtain follow-up data on patients, and we elected to include only complete data in order to report the current true results.

Although the pneumothorax rates were lower than many reported studies on CT-guided percutaneous lung biopsies, there was an overall chest tube rate of $12.4 \%$, which is higher than some reported studies in patients undergoing CT-guided biopsy. Our lower pneumothorax rate, in comparison to CT-guided biopsies, may be a function of imaging modality used to diagnose pneumothoraces. CT-guided biopsies verify the presence or absence of a pneumothorax using the CT scanner which is highly sensitive to diagnosing a pneumothorax, where the patients in this series were diagnosed using a combination of chest X-rays and thoracic ultrasound. Despite this, when adjusting for risk factors related to pneumothorax, we found that the highest volume single centre had a lower pneumothorax rate, $15 \%$ ( $7.6 \%$ chest tube rate), as compared to the entire study cohort. This leads us to believe that as centres perform more EM-guided percutaneous lung biopsies, there may be an overall decrease in pneumothorax rates. The authors also hypothesize that the clinicians in this study may also have had a lower 
threshold to place chest tubes in the setting of a newer technique in conjunction with the use of minimally invasive small-bore chest tubes (8Fr or $14 \mathrm{Fr}$ ) in all cases reported. No patients in the study suffered from a prolonged air leak.

In this study, we show that an EM-guided percutaneous lung biopsy approach yields a high diagnostic rate with minimal safety concerns and the added benefit of using a combined bronchoscopic and percutaneous staged approach to pulmonary nodule diagnostics in a single procedural setting. A prospective multicentre trial to validate these findings is currently underway (NCT03338049). ${ }^{21}$

\section{Acknowledgements}

Research reported in this publication was supported by the National Heart, Lung, and Blood Institute of the National Institutes of Health under Award Number T32HL007534. The content is solely the responsibility of the authors and does not necessarily represent the official views of the National Institutes of Health.

\section{Disclosure statement}

H.L., J.A., H.B., G.S., A.C. and L.Y. have received educational grants and/or consulting fees from Veran Medical. This study was previously presented at the 2017 CHEST Annual Meeting.

Abbreviations: BMI, body mass index; COPD, chronic obstructive pulmonary disease; CPT, current Procedural terminology; CT, computed tomography; EBUS, endobronchial ultrasound; EM, electromagnetic; FNA, fine needle aspiration; ROSE, rapid on-site evaluation; SPN, solitary pulmonary nodule.

\section{REFERENCES}

1 Aberle DR, Adams AM, Berg CD, Black WC, Clapp JD, Fagerstrom RM, Gareen IF, Gatsonis C, Marcus PM, Sicks JD. Reduced lung-cancer mortality with low-dose computed tomographic screening. N. Engl. J. Med. 2011; 365: 395-409.

2 Ma J, Ward EM, Smith R, Jemal A. Annual number of lung cancer deaths potentially avertable by screening in the United States. Cancer 2013; 119: 1381-5.

3 Mettler FA Jr, Thomadsen BR, Bhargavan M, Gilley DB, Gray JE, Lipoti JA, McCrohan J, Yoshizumi TT, Mahesh M. Medical radiation exposure in the U.S. in 2006: preliminary results. Health Phys. 2008; 95: 502-7.

4 Berrington de Gonzalez A, Mahesh M, Kim KP, Bhargavan M, Lewis R, Mettler F, Land C. Projected cancer risks from computed tomographic scans performed in the United States in 2007. Arch. Intern. Med. 2009; 169: 2071-7.

5 Gould MK, Tang T, Liu IL, Lee J, Zheng C, Danforth KN, Kosco AE, Di Fiore JL, Suh DE. Recent trends in the identification of incidental pulmonary nodules. Am. J. Respir. Crit. Care Med. 2015; 192: 1208-14.

6 Moyer VA. Screening for lung cancer: U.S. Preventive Services Task Force recommendation statement. Ann. Intern. Med. 2014; 160: 330-8.

7 Naidich DP, Bankier AA, MacMahon H, Schaefer-Prokop CM, Pistolesi M, Goo JM, Macchiarini P, Crapo JD, Herold CJ, Austin JH et al. Recommendations for the management of subsolid pulmonary nodules detected at CT: a statement from the Fleischner Society. Radiology 2013; 266: 304-17.

8 Krochmal R, Arias S, Yarmus L, Feller-Kopman D, Lee H. Diagnosis and management of pulmonary nodules. Expert Rev. Respir. Med. 2014; 8: 677-91.

9 Wiener R, Schwartz LM, Woloshin S, Welch H. Population-based risk for complications after transthoracic needle lung biopsy of a pulmonary nodule: an analysis of discharge records. Ann. Intern. Med. 2011; 155: 137-44.

10 Facciolongo N, Patelli M, Gasparini S, Lazzari Agli L, Salio M, Simonassi C, Del Prato B, Zanoni P. Incidence of complications in bronchoscopy. Multicentre prospective study of 20,986 bronchoscopies. Monaldi Arch. Chest Dis. 2009; 71: 8-14.

11 Schreiber G, McCrory DC. Performance characteristics of different modalities for diagnosis of suspected lung cancer: summary of published evidence. Chest 2003; 123: 115s-28s.

12 Yarmus LB, Arias S, Feller-Kopman D, Semaan R, Wang KP, Frimpong B, Oakjones Burgess K, Thompson R, Chen A, Ortiz R et al. Electromagnetic navigation transthoracic needle aspiration for the diagnosis of pulmonary nodules: a safety and feasibility pilot study. J. Thorac. Dis. 2016; 8: 186-94.

13 Larscheid RC, Thorpe PE, Scott WJ. Percutaneous transthoracic needle aspiration biopsy: a comprehensive review of its current role in the diagnosis and treatment of lung tumors. Chest 1998; 114: 704-9.

14 Loubeyre P, Copercini M, Dietrich PY. Percutaneous CT-guided multisampling core needle biopsy of thoracic lesions. AJR Am. J. Roentgenol. 2005; 185: 1294-8.

15 Loubeyre P, McKee TA, Copercini M, Rosset A, Dietrich PY. Diagnostic precision of image-guided multisampling core needle biopsy of suspected lymphomas in a primary care hospital. $\mathrm{Br}$. J. Cancer 2009; 100: 1771-6.

16 Heerink WJ, de Bock GH, de Jonge GJ, Groen HJ, Vliegenthart R, Oudkerk M. Complication rates of CT-guided transthoracic lung biopsy: meta-analysis. Eur. Radiol. 2017; 27: 138-48.

17 Harzheim D, Sterman D, Shah PL, Eberhardt R, Herth FJF. Bronchoscopic transparenchymal nodule access: feasibility and safety in an endoscopic unit. Respiration 2016; 91: 302-6.

18 Herth FJ, Eberhardt R, Sterman D, Silvestri GA, Hoffmann H, Shah PL. Bronchoscopic transparenchymal nodule access (BTPNA): first in human trial of a novel procedure for sampling solitary pulmonary nodules. Thorax 2015; 70: 326-32.

19 Sconfienza LM, Mauri G, Grossi F, Truini M, Serafini G, Sardanelli F, Murolo C. Pleural and peripheral lung lesions: comparison of US- and CT-guided biopsy. Radiology 2013; 266: 930-5.

20 Lee H, Lerner AD, Coleman B, Semaan R, Mallow C, Arias S, Salwen B, Feller-Kopman D, Yarmus L. Learning electromagnetic navigational bronchoscopy and percutaneous transthoracic needle biopsy (LEAP): a pilot study. J. Bronchology Interv. Pulmonol. 2019; 26: $55-61$.

21 Thiboutot J, Lee HJ, Silvestri GA, Chen A, Wahidi MM, Gilbert CR, Pastis NJ, Los J, Barriere AM, Mallow C et al. Study design and rationale: a multicenter, prospective trial of electromagnetic Bronchoscopic and electromagnetic transthoracic navigational approaches for the Biopsy of peripheral pulmonary nodules (ALL IN ONE Trial). Contemp. Clin. Trials 2018; 71: 88-95.

\section{Supplementary Information}

Additional supplementary information can be accessed via the $h \mathrm{tm} /$ version of this article at the publisher's website.

Visual Abstract Electromagnetic guided transthoracic needle biopsy is safe, feasible and has high diagnostic yield 